\title{
TWO APPLICATIONS OF DEVELOPMENTAL GENETICS TO PALEONTOLOGY: SEGMENTATION GENES IN MOLLUSCS AND PREORAL APPENDAGES IN TAXA OF UNCERTAIN AFFINITY
}

\author{
JACOBS, David K., Dept of Invertebrates, American Museum of Natural History,
} Central Park West at 79th Street, New York, NY 10024-5192.

Resolution of deep evolutionary problems, including the origin of the metazoa and the morphologic evolution of higher taxa within the metazoa, have long been sought in the developmental sequence. Haeckel's gastraea theory is perhaps the best example of this endeavor. Since Haeckel's time it has become apparent that the early evolution of animal life cannot be read directly from the developmental sequence. Ontogeny itself evolves making it difficult to even identify homologies in the early development between many phyla and classes. However, all may not be lost; during metazoan development gene expression must be localized in order to differentiate cell types in the body during development. It is this regionalized transcription and translation to protein product that differentiates cell types, organ systems, and the morphologic features that we can identify in the fossil record. The functional importance of the genes in question, and the fact that portions of the protein products of these genes must bind to DNA in order to perform their switching function, leads to extreme sequence conservation. This permits the identification and comparison of homologous genes important in the development of divergent taxa even after the passage of the entire Phanerozoic. If these genes retain a pattern of expression in development as well as conservation of the DNA sequence, then we can identify a homologous process derived from the development of the shared ancestor of the two taxa.

This approach can be used to address the homology of metameric units. Preliminary results indicate that the segmentation gene engrailed is expressed in chiton trochophores in associated with each of the developing plate fields. The engrailed gene is known to control segmentation processes in arthropods and annelids. Evidently the plates in chitons evolved from serial features in a shared ancestor of annelids, arthropods and molluscs. This indicates that the serial features found in molluscs are ancestral (plesiomorphic), and that the evolution of the mollusca has involved the loss or reduction of serial features rather than their de novo evolution in chitons and monoplacophorans as has been suggested by a number of neontologists. The fossil record suggests successive reduction of serial features in molluscs. This paleontological interpretation now finds support in developmental genetics.

Developmental genetics also provides a basis for evolution of body plans. Unusual preoral appendages have evolved in chelicerates, Burgess Shale arthropods such as Yohoia and Leanchoilia, and non arthropod forms such as Opabinia and Tullimonstrum. The differentiation of the vertebral column of vertebrates and the segments of arthropods are controlled by homologous genes that are expressed in a anterior to posterior sequence. Out of place (ectopic) expression of posterior genes in the anterior region produces posterior features in the antennal field of arthropods and in the most anterior vertebrae of the vertebrates. This potential for conversion of anterior features was present in the shared ancestor of vertebrates and arthropods. Thus evolution of novel anterior features resulting from ectopic expression could occur in both deuterostomes and protostomes and may account for a range of novel fossil forms. This suggests that parallel evolution of unusual anterior features could occur, and the presence of these features may not be the best character to use in a phylogenetic analyses. 